\title{
From Antimicrobial Activity to Mechanism of Resistance: The Multifaceted Role of Simple Quaternary Ammonium Compounds in Bacterial Eradication
}

\author{
Kevin P.C. Minbiole ${ }^{\mathrm{a}}{ }^{*}$ Megan C. Jennings $^{\mathrm{b}}$, Laura E. Ator ${ }^{\mathrm{a}}$, Jacob W. Black ${ }^{\mathrm{a}}$, Melissa C. Grenier ${ }^{\mathrm{a}}$, Jade E. \\ LaDow $^{\mathrm{c}}$, Kevin L. Caran ${ }^{\mathrm{d}}$, Kyle Seifert ${ }^{\mathrm{c}}$, and William M. Wuest ${ }^{\mathrm{b}} *$ \\ ${ }^{a}$ Department of Chemistry, Villanova University, 800 Lancaster Avenue, Villanova, PA 19085, United States \\ ${ }^{b}$ Department of Chemistry, Temple University, $1901 N 13^{\text {th }}$ Street, Philadelphia, PA 19122, United States \\ ${ }^{c}$ Department of Biology, James Madison University, 951 Carrier Drive, MSC 7801, Harrisonburg, VA 22807, United States \\ ${ }^{d}$ Department of Chemistry and Biochemistry, James Madison University, 901 Carrier Drive, MSC 4501, Harrisonburg, VA 22807, United States
}

ARTICLE INFO

Article history:

Received

Received in revised form

Accepted

Available online

Keywords:

Quaternary ammonium compound

Antibacterial

Antibiofilm

Amphiphile

MultiQAC

\section{ABSTRACT}

Quaternary ammonium compounds (QACs) are a prominent class of antibacterial agents. Drawing inspiration from commercial disinfectants and antimicrobial natural products, we have derivatized structurally diverse tertiary amines to generate several different classes of QACs. We have synthesized over 200 QACs, many of which exhibit potent antibacterial and antibiofilm activity. Analysis of the structure-activity relationship of our compounds have led to the facile production of inexpensive QACs that display $\sim 1 \mu \mathrm{M}$ MIC against a suite of bacteria, and furthermore do not appear to trigger bacterial resistance systems in methicillin-resistant Staphylococcus aureus (MRSA).

2009 Elsevier Ltd. All rights reserved.

\section{Introduction}

Pathogenic bacteria have long plagued humans and are consistently among the foremost health crises worldwide. While we are accustomed to repelling the invasion of such pathogens with both innate defenses as well as medical protections, many bacteria that are encountered evade our immunities and are resistant to multiple antibiotics of both natural and synthetic origin. ${ }^{1,2}$ Bacterial infections lead to hundreds of thousands of deaths and billions of dollars in healthcare costs annually, necessitating a constant push to discover and improve strategies to counter these threats. ${ }^{3,4}$ Furthermore, bacterial biofilms that exist outside of the body on abiotic surfaces pose severe consequences, including biocorrosion and biofouling, across a range of industries. ${ }^{5}$ Such detrimental biofilm processes can contribute toward the contamination of drinking water and industrial products, increased maintenance costs, and destruction of essential equipment.
One prominent and critical class of antibacterials is the quaternary ammonium compounds (QACs). ${ }^{6}$ QACs with permanent positive charges are found in antimicrobial natural products such as berberine, ${ }^{7}$ whereas tertiary nitrogens in polyamines such as norspermidine provide examples of $\mathrm{pH}$ dependent QACs (Figure 1). ${ }^{8}$ Synthetic amphiphilic QACs are among the most heavily produced and utilized commercial products and have found use as surfactants, phase transfer catalysts, and disinfectants ${ }^{1,9}$ - the latter of which will be the focus of this report. Though initial reports date back to the early $1900 \mathrm{~s},{ }^{10}$ QACs first gained traction in the disinfectant market in the 1930s and 1940s with the approval of benzalkonium chloride as an antimicrobial agent. ${ }^{11}$ Since the dawn of this class of antimicrobials, several QACs have been marketed as antibacterials for use in several arenas including hospitals and healthcare facilities, various industries, and commercial and residential settings. A sampling of QACs currently on the market are depicted in Figure 1 and include benzalkonium chloride

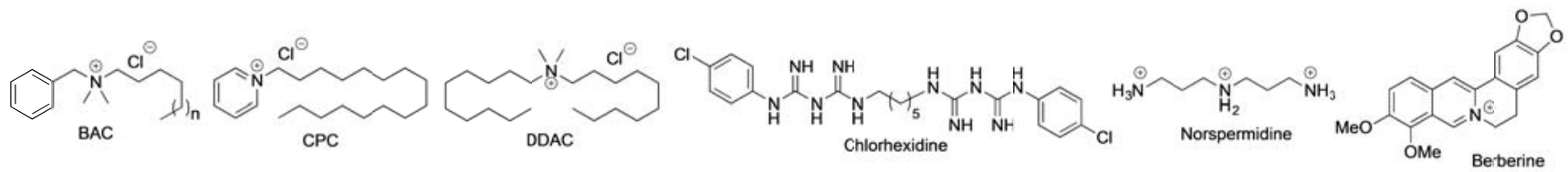

Figure 1. Commercially available and naturally occurring quaternary ammonium compounds.

* Corresponding author. Tel.: +1-610-519-4873; fax: +1-610-519-

7167; e-mail: kevin.minbiole@villanova.edu

(C) 2016. This manuscript version is made available under the Elsevier user license http://www.elsevier.com/open-access/userlicense/1.0/ 
(BAC), cetylpyridinium chloride (CPC), didecyldimethylammonium chloride (DDAC), and chlorhexidine (CHX). In examining the structures of commercial QACs, it is apparent that most are monocationic derivatives with little structural variability, thus making this an area ripe with opportunity for expansion and investigation.

Of significant concern, reports of bacteria resistant to a battery of commercial QAC disinfectants have been rampant in recent years. ${ }^{6}$ This defense mechanism, which was originally attributed to the efflux of antimicrobial natural products such as berberine, has further been characterized through QAC-related dyes. Resistance occurs via QAC-specific efflux pumps such as QacA, the transcription of which is regulated by DNA-binding regulator QacR. ${ }^{6}$ Herein we report the culmination of over five years of research that started as a straightforward structureactivity relationship study of QAC scaffolds as antimicrobials and has since shifted toward using this library to elucidate the mechanism of QAC-resistance.

\subsection{BisQACs}

The impetus of this research program began with our vision to explore the utility of QACs that bore more than one positive charge, in contrast to the commercially available agents. In addition, we wanted to focus on maintaining flexible synthetic routes that would allow for significant structural variation. Multicationic QACs were viewed as synthetically accessible targets that would possess a fundamentally different interaction with bacterial cell membranes, the long-assumed target of amphiphilic antiseptics. ${ }^{6}$ We initially aimed to incorporate an unequal ratio of quaternary centers to long alkyl chains, hypothesizing that bicephalic (two-headed) amphiphiles with just one non-polar tail would possess superior membrane disruption capability, owing to the high cone angles of their structures. ${ }^{12}$ As we moved forward, we turned our focus to another design element - incorporating core structures that were commercially available at an economical cost and possessed at least two tertiary amines that could be readily quaternized. The evolution of our work toward multiQACs, both failures and successes, are described in this section, and an overview is presented in Scheme 1.

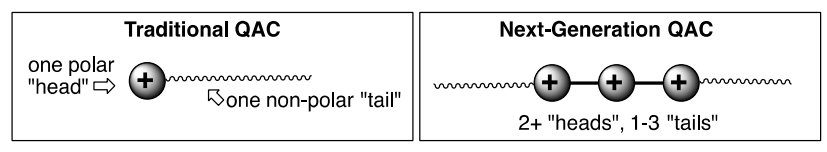

Scheme 1. Next generation QAC structures developed in our laboratories.

\subsubsection{Aryl bisQACs - Initial discoveries}

With clear analogy to the commercial antibacterial QAC benzalkonium chloride, our endeavor began with the investigation of bicephalic aryl amphiphiles connected via a benzyl linker. ${ }^{13}$ In a three-step route, a series of twelve bistrimethylammonium aryl ethers were synthesized (Figure 2).
Specifically, a variety of methyl-substituted phenols were Oalkylated, brominated at the benzylic position, and then exhaustively reacted with trimethylamine, furnishing a series of twelve bisQACs bearing a single alkyl chain. These were tested for minimum inhibitory concentration (MIC) against a standard panel of four bacteria, ${ }^{14}$ including two Gram-positive (Staphylococcus aureus and Enterococcus faecalis) and two Gram-negative (Pseudomonas aeruginosa and Escherichia coli) strains. While all amphiphiles of this series were able to kill at least two of four bacterial strains, two compounds, 2,4-C14 and $3,5-\mathrm{C} 14$, were identified as being the most effective. Our results indicated that biscationic amphiphiles with longer $(C>12)$ alkyl chain lengths were most effective at killing bacteria, while the relative position of the quaternary ammonium groups accounted for a $\leq 4$-fold difference in antibacterial activity. Further, while activities were good (MICs for 3,5-C14 ranged from 4-63 $\mu \mathrm{M}$, as highlighted in the box in Figure 2), the potency of these bicephalic amphiphiles was not superior to other known compounds. A key unpublished result, however, identified a structural modification that showed promise: replacement of the trimethylammonium group with a pyridinium group led to enhanced activity in some cases, such as in the 2,3 series (Figure 2, right; manuscript in preparation). For comparisons of antimicrobial activity across multiple structural classes, the reader is both pointed towards Table 1 for a list of our most potent antimicrobials, as well as exhaustive MIC table (237 compounds) presented in the Supplementary Material.

\subsubsection{Aryl bisQACs - 4,4'-Bipyridinium (paraquat) compounds}

Taking a cue from the promising pyridinium analogs of the aryl alkoxy compounds above, we moved to an investigation of the antibacterial activity of 4,4'-bipyridinium amphiphiles. ${ }^{15}$ Given the known redox properties of the herbicide $N, N^{\prime}$ dimethyl-4,4'-bipyridinium dichloride, commonly called paraquat, this inexpensive commercially available scaffold was a logical choice for exploring a broad series of quaternized derivatives. In one or two steps, a set of 36 paraquat (PQ) amphiphiles bearing alkyl side chains of various lengths were synthesized (Figure 3). In addition to exploring the effect that alkyl side chain length had on antibacterial activity, the influences of counter ions and asymmetry on activity were also explored. The most potent amphiphiles, PQ-11,11 and PQ-12,10, exhibited MICs as low as $2 \mu \mathrm{M}$ against three of the four bacteria tested; amphiphiles with slightly increased asymmetry, such as PQ-14,8, showed nearly equipotent values, while more significant asymmetry was found to be detrimental to antibacterial activity. Further, variations in counter ion nature were found to be negligible in the shorter-chained compounds; however, the iodide counter ion did severely hamper water solubility, and therefore bioactivity, in chain lengths exceeding twelve carbons.

\subsubsection{Aryl bisQACs - Alternative pyridinium structures}

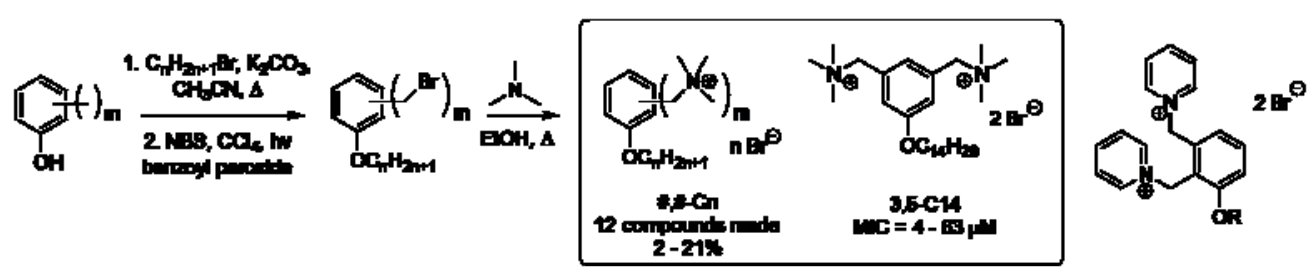

Figure 2. Bicephalic QACs: representative syntheses of bis-trimethylammonium aryl alkoxy QACs, and pyridinium analogs. ${ }^{13}$ 


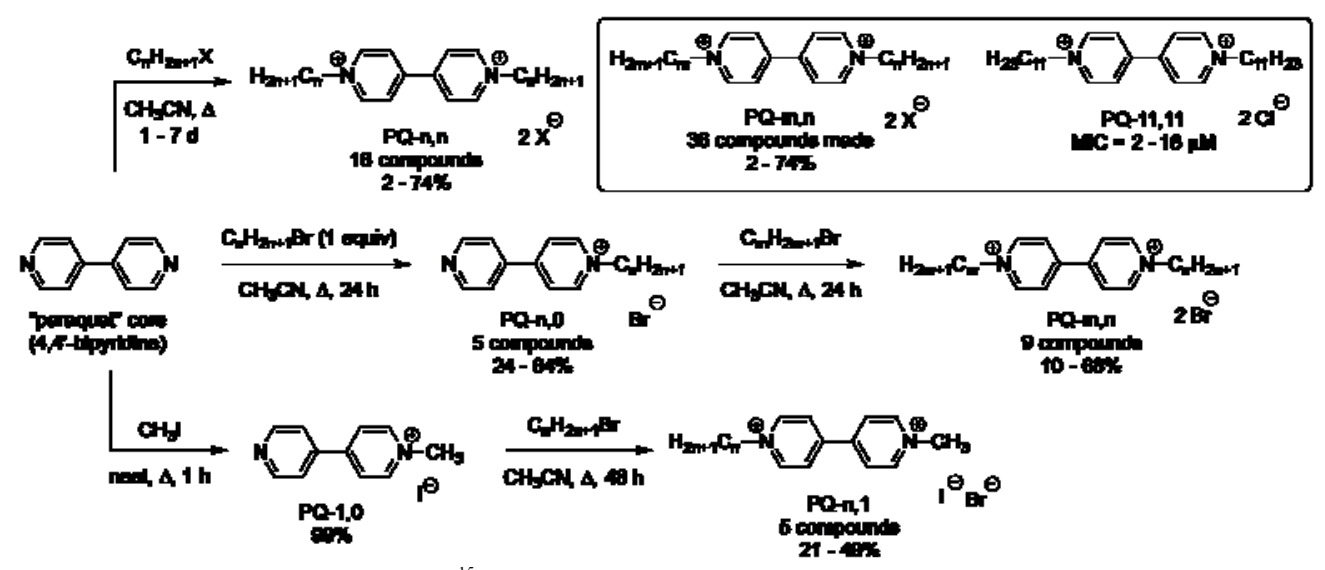

Figure 3. Bis-pyridinium QACs: the paraquat series. ${ }^{15}$

While quaternized paraquats were found to be potent antibacterial compounds, their known redox activity also augments their toxicity to eukaryotic cells via generation of superoxide. ${ }^{16}$ In order to attenuate this shortcoming, we sought to explore other bipyridine isomer scaffolds, including the common 2,2'-bipyridinium ligand (orthoquat, $O Q$ ) and the affordable 2,4'bipyridinium core (paraorthoquat, POQ). Unfortunately, these two bipyridine isomers were not converted to bisQACs under our standard alkylation conditions. In the case of OQ, the basicity of the proximal nitrogen atoms was the presumable cause of failure, as we saw little alkylation (Figure 4, top). Further, we were not successful in bis-alkylating 2,4'-bipyridine, presumably due to the poor reactivity of the "ortho"-nitrogen (Figure 4, bottom). Only two monoQAC derivatives (POQ-12,0 and POQ-18,0) were prepared; POQ-12,0 had moderate activity, and POQ-18,0 was not tested due to poor water solubility.
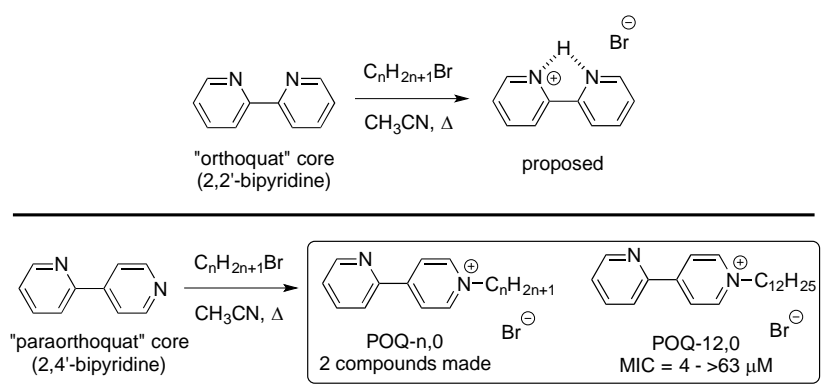

Figure 4. Investigations into alternative bipyridine systems: orthoquat and paraorthoquat derivatives.

Undeterred, we shifted our efforts towards the preparation of 3,3'-bipyridine (metaquat, MQ) and 3,4'-bipyridine (parametaquat, PMQ), as such scaffolds were thought to possess redox properties distinct from their paraquat isomer and be synthetically accessible. ${ }^{17}$ Due to significantly increased cost of 3,3'-bipyridine as compared to its 4,4'-bipyridine isomer, we initially set out to synthesize 3,3'-bipyridine via an aryl halide homocoupling reaction. Our initial coupling reaction failed to proceed, and this fact initially eluded our detection; we thus alkylated the recovered $m$-bromopyridine and unintentionally prepared a small set of "meta-bromopyridinium" (MBP) compounds (Figure 5). While exhibiting some inhibitory activity against Gram-positive bacteria, this series proved ineffective in inhibiting growth of Gram-negative bacteria (MIC vs PA: 400 $\mu \mathrm{M})$. This result, along with the analysis of POQ-12,0, served as confirmation that our biscationic compounds showed greater promise than monocationic analogs.

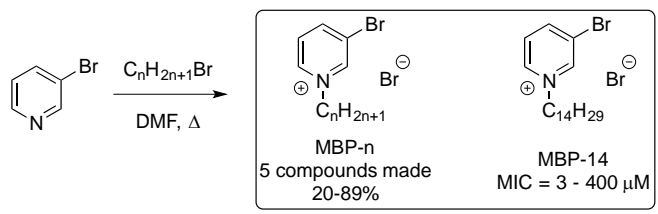

Figure 5. Other pyridinium structures: $m$-bromopyridinium QACs.

Palladium-mediated cross-coupling of either 3- or 4-pyridyl boronic acid with 3-bromo pyridine led to 3,3'-bipyridine and $3,4^{\prime}$-bipyridine, respectively. ${ }^{18}$ Subsequently, in one or two additional steps, a series of eleven MQ and six PMQ amphiphiles were prepared using standard alkylation conditions (Figure 6). ${ }^{17}$ Inspired by the PQ series, a small, representative series of asymmetric MQs were included; however, asymmetry had no significant positive effect on antibacterial activity. In fact, consistent with the PQ series, the most potent amphiphiles were 
those with 22-24 total side-chain carbons, with MQ-11,11 and PMQ-11,11 displaying MIC values as low as $0.5 \mu \mathrm{M}$ against the tested bacteria. Ultimately, we concluded that MQ and PMQbased bisQACs are as active - if not more active - against all bacterial strains tested as compared to their analogous PQ-based amphiphiles. And although we saw significantly diminished redox activity in the MQ and PMQ series, toxicity studies showed that all three bipyridyl amphiphile series display identical hemolysis values $(8 \mu \mathrm{M}$ for PQ-12,12, PMQ-12,12, and MQ12,12). ${ }^{19}$ This ended our efforts to mitigate bispyridinium toxicity by altering the redox properties of the scaffold.

\subsubsection{Assembly of QACs with “click” chemistry}

In light of our successes in preparation of bis-heterocyclic bisQACs, we aimed to extend our synthetic strategies to include click chemistry, wherein rapid and atom-economical reactions could introduce another nitrogenated heterocycle, the 1,2,3triazole. Accordingly, we utilized 3-ethynylpyridine in a coppermediated click reaction, using either in-situ generated decyl, undecyl, or tetradecyl azide; this furnished meta-substituted pyridinium triazoles (abbreviated $\mathrm{PmT}_{\mathrm{m}}$ ) shown in Figure 7. To our disappointment, however, bisalkylation was not effected, as we could only assemble the monoQACs shown in Figure 7. And, since harsh methylation conditions $\left(\mathrm{MeI}\right.$ or $\left.\mathrm{Me}_{2} \mathrm{SO}_{4}\right)$ did not affect a second alkylation at the triazole, and our above investigations showed that aryl monoQACs showed only modest activity, this route was not carried further.

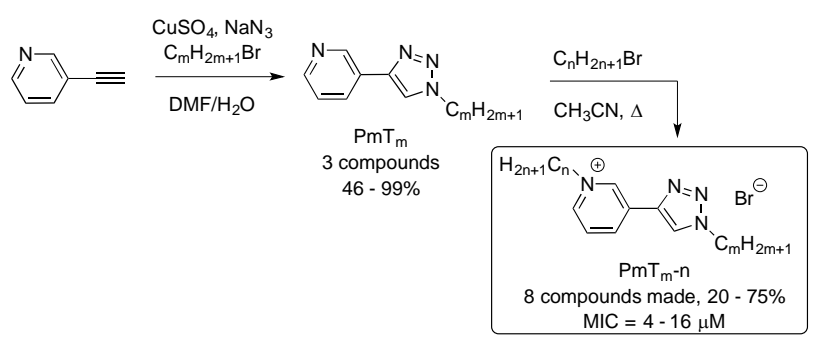

Figure 7. $m$-Pyridinium-triazole monoQACs generated by "click" chemistry and alkylation.

\subsubsection{Alkyl bisQACs}

Concurrent with our investigations into aryl bisQACs, we pursued a distinct class of QACs derived from commercially available structures possessing multiple tertiary amines on an alkyl backbone. ${ }^{20}$ One highly promising scaffold presented itself in the cost-effective tetramethylethylenediamine (TMEDA), whose symmetrical alkylation was well precedented; ${ }^{21,22,23}$ however, little study of the antimicrobial activity of TMEDAbased QACs had been reported. ${ }^{24,25}$ Thus TMEDA was alkylated both symmetrically and asymmetrically to yield a collection of 36 TMEDA-derived mono- and bisQACs (Figure 8). ${ }^{20}$ When subjected to our standard panel of bacteria, bioactivity data supported the observations from our aromatic compound sets: compounds bearing a high degree of symmetry as well as those comprised of a total of 20-24 side chain carbons possessed optimal bioactivity. To our delight, multiple TMEDA amphiphiles exhibited single-digit micromolar MIC values for all four bacteria. This economical alkyl scaffold allowed for efficient preparation of bisQAC amphiphiles, providing an encouraging introduction to our next endeavors developing more complex polyamine QACs.

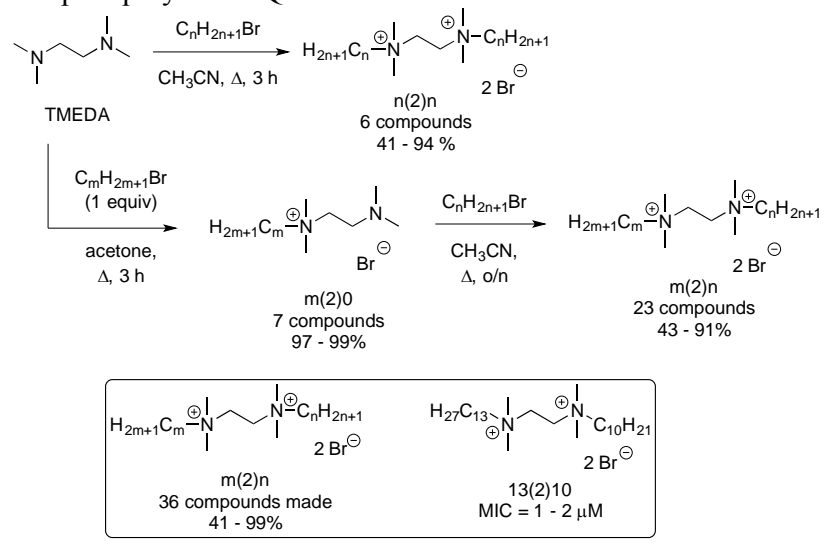

Figure 8. Symmetric and asymmetric TMEDA-derived QACs. ${ }^{20}$

\subsubsection{Natural products as core structures for bisQACs - Alkyl/aryl QACs}

Having found success in employing both aromatic and alkyl amine cores for functionalization into QAC structures, we decided to investigate an alternate starting platform for QAC assembly - natural products. Two inexpensive structures quickly came to light: quinine and nicotine. These formed a complementary set, in that both possess a heteroaromatic nitrogen as well as a tertiary alkyl amine. They differ, however, in which of these nitrogen atoms is the most easily alkylated quinine is more reactive at the tertiary amine of the bicyclic system, ${ }^{26}$ and nicotine is preferentially alkylated at the pyridine moiety. ${ }^{27}$ Such preferences were exploited in the synthesis of the bisQACs shown below (Figure 9). To our surprise, results from these scaffolds showed monoQACs of equal or superior activity to their bisQAC analogs; in fact, our most potent compound in this series was the monoalkylated quinine derivative Q-14,0.

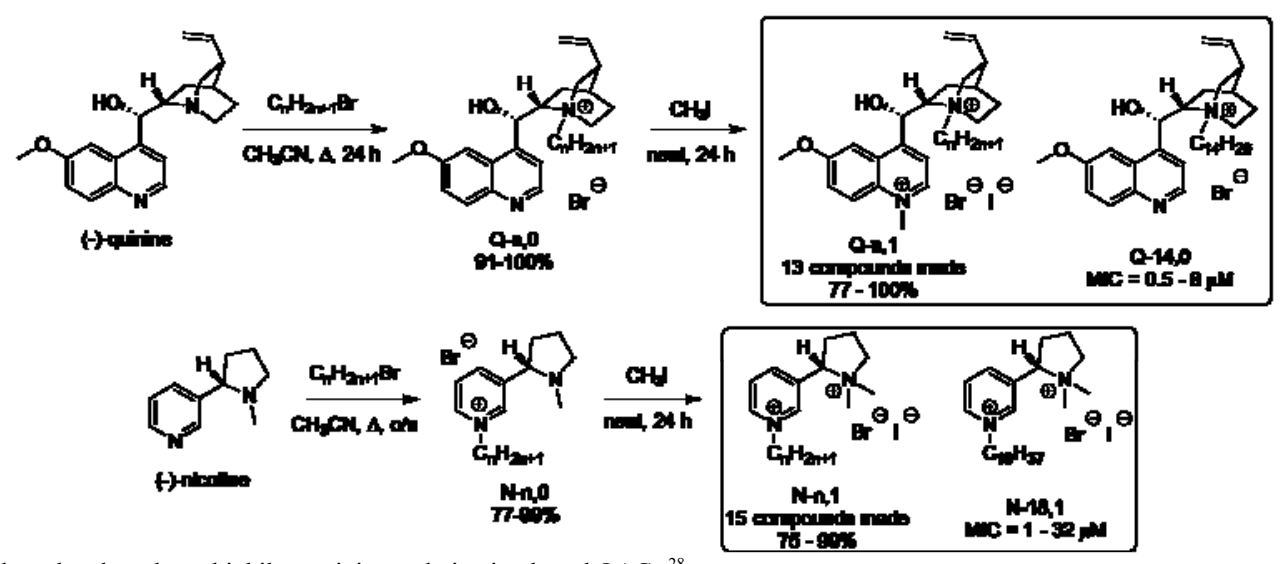

Figure 9. Natural product based amphiphiles: quinine and nicotine-based QACs. ${ }^{28}$ 
While we did not unearth any compounds as potent as the ones described above, we were pleased to develop a distinct approach to the construction of novel QAC architectures. ${ }^{28}$

\subsection{MultiQACs}

QACs can be viewed as synthetic analogs of antimicrobial peptides - general purpose amphiphiles designed to provide an initial level of protection against pathogenic invaders arriving to a surface, be it the skin of an organism or the countertop of a kitchen. And the natural amphiphiles present multiple charged groups in addition to many non-polar sections. Having found success in both the facile preparation and the powerful antimicrobial activity of the alkyl and aryl bisQAC structures described above, we chose to pursue compounds with three or four cations. Our campaign of antibacterial multiQACs was partially inspired by work suggesting that polyamines, such as norspermidine (Figure 1), are involved in biofilm dispersion. ${ }^{8}$ Based on initial reports, we hypothesized that combining the antibacterial characteristics of QACs with the biofilm dispersion effects of polyamines would lead to a novel class of potent biofilm eradicators. ${ }^{9}$ Beginning with an extended polyamine backbone, we aimed to construct multiQACs to examine disinfectant properties against both planktonic and biofilm bacteria.

\subsubsection{Linear alkyl multiQACs}

As we transitioned towards QACs with greater overall charge, our strategy of identifying inexpensive core structures was again successful; structures bearing three tertiary amines were available from standard commercial sources (i.e., Figure 10). Alkylation of these structures was found to exclusively take place at the terminal amines, which allowed for differential substitution (or lack of substitution) at the central nitrogen to probe the role that the number of cations plays in antibacterial behavior. ${ }^{29} \mathrm{~A}$ range of analogs, some bearing alkene and thiol groups, were installed (shown as $\mathrm{R}$ in Figure 10), providing opportunity to use these groups as "handles" for further functionalization. Data from this study confirmed our previous findings that side chains of eleven to fourteen carbons were generally optimal; those QACs bearing shorter chains extending from the terminal nitrogens were less active. A tetraamine substrate was also employed and was likewise readily alkylated at the terminal nitrgens with good selectivity; this was followed by allylation or methylation at the center nitrogens. Subsequent antibacterial activity determinations held a surprise: there was no significant advantage in the installation of a fourth nitrogen atom, either as the neutral amine or quaternized as the tetraQAC.
In addition to testing against Gram-positive and Gramnegative planktonic bacteria, we wished to test our hypothesis that alkyl multiQACs would be capable of biofilm eradication. In such an assay, pre-established mature biofilms are subjected to QAC treatment and resulting eradication of the biofilm is measured and reported as the minimum biofilm eradication concentration (MBEC). ${ }^{9,19}$ Given the transient nature of biofilms, such assays are notoriously difficult to reproduce. We were therefore delighted to uncover strong MBEC values (as low as 25 $\mu \mathrm{M})^{9}$ against bacterial biofilms, including MRSA biofilms. ${ }^{19} \mathrm{We}$ were further satisfied that one of our compounds, 12(2)1(2)12, has in fact been used in other laboratories as a positive control for biofilm eradication. ${ }^{30}$ In our own studies, we discovered that while cationic or polyamine nature alone is not capable of biofilm disruption, our alkyl multiQACs are among the most efficacious biofilm eradicators published to date.

\subsubsection{Scaffold hopping}

Having investigated a number of linear multiQACs, as well as a series of aromatic-based multiQACs, we chose to enhance structural variety by adopting a scaffold-hopping approach to the construction of multiQACs. ${ }^{31}$ Commercial sources offered three alternative scaffolds presenting either cyclized or branched structures bearing three or four tertiary amines. We hoped to uncover an optimal disposure of the alkyl groups to better perturb the inegrity of bacterial cell membranes; molecular modeling supported this idea. Thus, alkylation was pursued as shown in Figure 11. For the piperazine or "P-series", we again found that alkylation was readily achieved at the peripheral nitrogens; however, alkylation was not effected at the center nitrogen, even under forcing conditions. The "C-series," bearing a cyclononyl ring, led to monoalkylation in high yield with a long-chained electrophile and only methylation (iodomethane) at the other positions. Finally, the "T-series," with the branched T-shaped core structure shown, allowed for trisalkylation in quite high yields, but again, left the central nitrogen unavailable for further alkylation. Biological highlights included the highly active compound T-11,11,11, which showed an MIC of $0.5-1 \mu \mathrm{M}$ against all bacteria but $P A$, where activity was $2 \mu \mathrm{M}$. Additionally, we noted some significant differentiation of activity in select compounds when facing methicillin-susceptible Staphylococcus aureus (MSSA) as opposed to methicillinresistant Staphylococcus aureus (MRSA), which will be elaborated upon in Section 3.

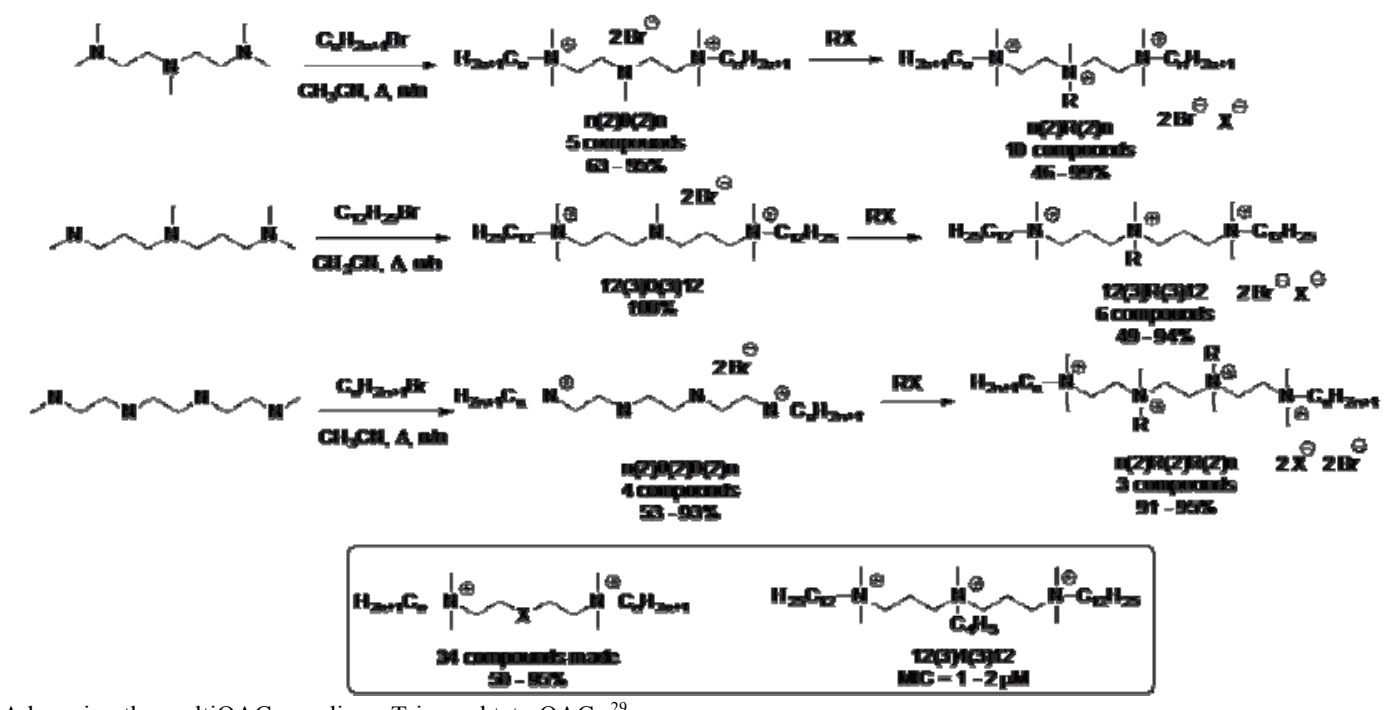

Figure 10. Advancing the multiQAC paradigm: Tris- and tetraQACs. ${ }^{29}$ 


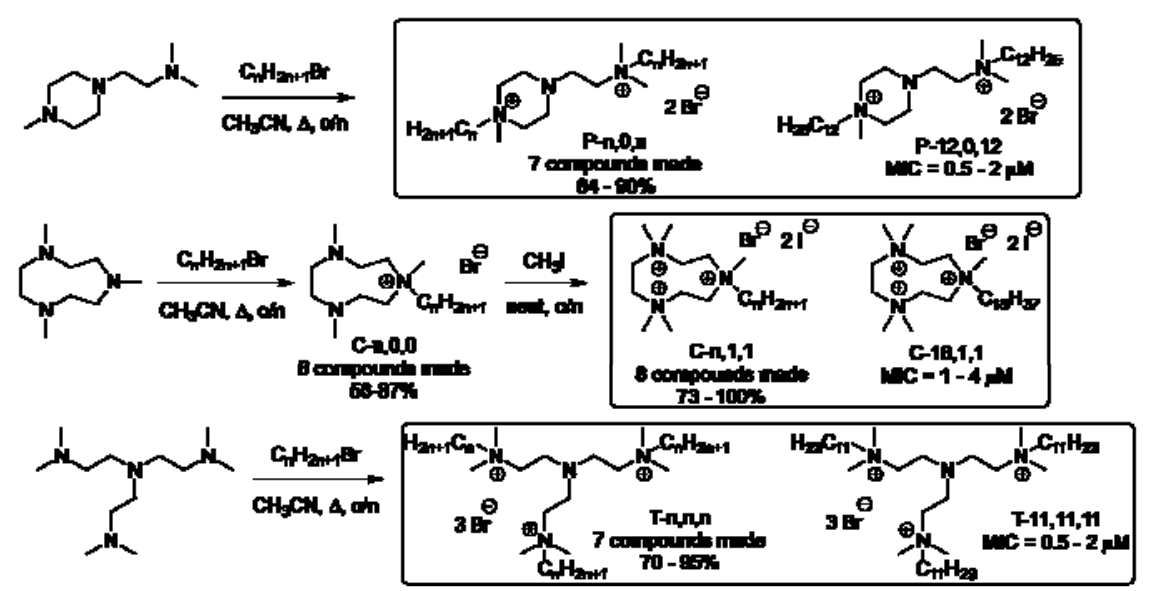

Figure 11. Scaffold hopping: use of cyclic and branched multiamine starting structures to produce varied QAC architectures. ${ }^{31}$

In order to broaden our investigations into alternative scaffolds, as well as the effect of the number and identity of cations and the number of tails on the antibacterial activity of multiQACs, two series of amphiphiles - each with three quaternary ammonium groups and two tails connected to a central arene core - were prepared (Figure 12). ${ }^{32}$ Monoalkylation of a symmetrical tris-benzylbromide (TBB) was accomplished using either pyridine or trimethylamine, and subsequent reaction with an excess of alkyldimethylamine afforded the target products. For each series, the compound with two 12-carbon chains had the strongest antibacterial activity, with MIC values of 1-2 $\mu \mathrm{M}$ for Gram positive and 4-16 $\mu \mathrm{M}$ for Gram-negative bacteria. Interestingly, for these series, no significant differences were found between the series with trimethylammonium (M$1, n, n)$ or pyridinium $(M-P, n, n)$ groups.
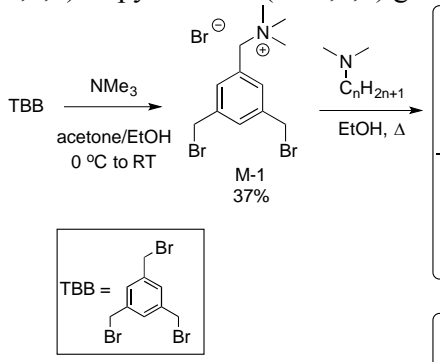

TBB

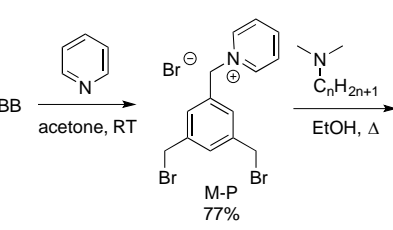

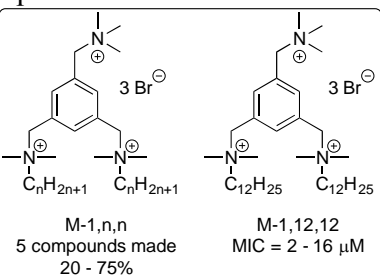

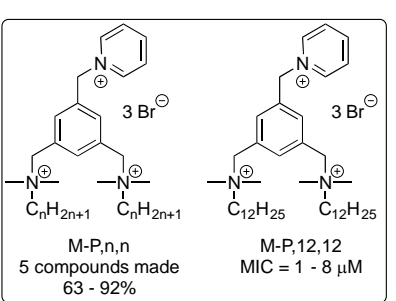

Figure 12. Three-headed two-tailed QAC structures. ${ }^{32}$

\subsection{Antimicrobial activity synopsis}

While the reader is referred to the supporting information for an exhaustive listing of the antimicrobial activity of the compounds presented above, Figure 13 and Table 1 present a synopsis of the "top compounds" prepared in our laboratories, regarding inhibition of our standard four-bacteria panel. Activity tends to peak for more symmetric compounds comprised of alkyl chains around twelve to fourteen carbons in length, which trends with the length required to penetrate and disrupt bacterial cell membranes. Furthermore, antibacterial activity does not necessarily increase with the number of cationic centers beyond bisQACs; activity tends to be more correlated with the number and length of the side chains appended to the quaternized nitrogens. From a scaffold point of view, there seems to be no inherent advantage to the antimicrobial activity of either aromatic or alkyl amine cores, and cyclic amines are likewise comparable to their linear counterparts. Finally, we note that some of our most rapidly assembled compounds (e.g., P-12,0,12, which is prepared in one high-yielding step) are amongst the most potent we have prepared.

Another theme emerges: our top compounds seem to plateau in activity, with a top MIC around $1 \mu \mathrm{M}$. Without a specific receptor or intracellular target, it seems that the bioactivity of amphiphilic QACs is directly proportional to their ability to disrupt microbial membranes. It follows then that QACs are generally more efficacious against Gram-positive bacteria as compared to Gram-negative bacteria, which possess a dual cell membrane. Interestingly, we found a significant increase in MIC of commercial monoQACs against methicillin-resistant Staphylococcus aureus (MRSA), leading us to explore the phenomenon of bacterial resistance to QACs.

We also note that the biofilm eradication activity of many of these compounds have been described elsewhere. ${ }^{9,29,31}$ Our more recent data ${ }^{31}$ suggest that our compounds do not demonstrate any specific capacity to destroy biofilms, i.e., via electrostatic interaction with extracellular polymeric substances. Presumably, the biofilm simply reduces the antimicrobial activity of the QACs. Accordingly, our compounds demonstrate reduced efficacy against MRSA biofilms, which are well known to resist chemical eradication.
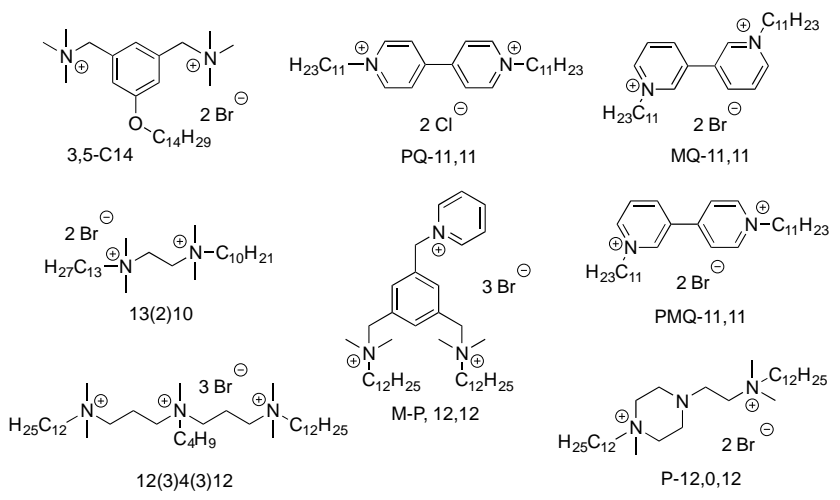

Figure 13. "Top compounds" produced in our laboratories.

Table 1. Minimum Inhibitory Concentrations (MIC) in $\mu \mathrm{M}$ of some best-in-class QACs.

Compound S. aureus E. faecalis E. coli $\quad$ P. aeruginosa 


\begin{tabular}{lcccc}
\hline $3,5-\mathrm{C} 14$ & 4 & 31 & 31 & 63 \\
PQ-11,11 & 2 & 2 & 2 & 8 \\
MQ-11,11 & 0.5 & 1 & 1 & 2 \\
PMQ-11,11 & 0.5 & 0.5 & 0.5 & 1 \\
$13(2) 10$ & 1 & 1 & 2 & 2 \\
$12(3) 4(3) 12$ & 1 & 1 & 1 & 2 \\
P-12,0,12 & 0.5 & 0.5 & 1 & 2 \\
M-P,12,12 & 2 & 2 & 4 & 8 \\
\hline
\end{tabular}

\subsection{QAC resistance}

Despite the long-held belief that QACs are virtually immune to resistance given their cell membrane target and non-specific mechanism of action, resistance to QACs has indeed been on the rise in recent years, especially among strains of MRSA. Resistance occurs via a class of closely-related efflux pumps, each specific to subset of QAC substrates. ${ }^{6}$ QacA and QacB are the most well-studied of such efflux pumps in Gram-positive bacteria, and QacR is the transcription regulator common to all of these systems. While it is known that certain classes of monoand bisQACs - including BAC, chlorhexidine, biguanides, dyes, and natural products such as berberine - are recognized by QacA/QacB and QacR, little work has been conducted on alternate QAC scaffolds or on QACs possessing higher charge.

We envisioned further exploring this arena through chemical biology; that is, designing compounds to test several aspects of this resistance system. In order to probe our hypothesis that QACs of higher charge state are not susceptible to current QAC resistance machinery, we utilized a variety of our bis, tris-, and tetraQACs as chemical probes against a strain of QACsusceptible $S$. aureus (MSSA) and a strain of $S$. aureus known to be resistant to QACs (MRSA). We found that while the majority of our QACs displayed no difference in activity between the two strains, those possessing a lower charge state and those with greater conformational rigidity (i.e., the $\mathrm{C}$-series) displayed the greatest disparity in activity between MSSA and MRSA. ${ }^{31}$ We furthermore noted that only those compounds containing aryl moieties exhibited resistance, providing insight as to the recognition of QACs in the QAC resistance machinery.

To determine the relative rate of resistance development, we subjected MSSA and MRSA to continuous sub-lethal doses of a variety of QACs including several commercial monoQACs and several of our bis-, tris-, and tetraQACs. ${ }^{19}$ Our results (Table 2) show that MSSA developed de novo resistance to commercial

\section{Experimental section}

All reactions were carried out under ambient atmosphere with reagent grade solvents and magnetic stirring. All reagents were purchased from commercial vendors. Yields refer to spectroscopically pure compounds. ${ }^{1} \mathrm{H}-\mathrm{NMR}$ spectra were recorded on a Varian Mercury 300 spectrometer. Chemical shifts are reported in $\delta$ values relative to tetramethylsilane. The reader is referred to the experimental detail for approximately 200 compounds, provided in references $13,15,17,19,20,28,29,31$, and 32 .

\subsection{3-(1-decyl-1H-1,2,3-triazol-4-yl)pyridine $\left(\mathrm{PmT}_{10}\right)$}

To a solution of 3-ethynylpyridine $(0.201 \mathrm{~g}, 2.91 \mathrm{mmol})$ in a solvent mixture of dimethylformamide and water $(4: 1,5 \mathrm{~mL})$ was added sodium ascorbate $(0.15 \mathrm{~g}, 0.75 \mathrm{mmol})$, sodium azide $(0.291 \mathrm{~g}, 1.47 \mathrm{mmol})$, decylbromide $(0.610 \mathrm{~mL}, 2.91 \mathrm{mmol})$ and
monoQACs as well as a novel aryl-containing bisQAC within a few hundred generations; no change in activity - indicating a lack of resistance - was observed for QACs of higher charge. Taken together, these results provide evidence that tris- and tetraQACs may be a viable option through which to pursue next-generation antibacterial agents.

Table 2. Development of resistance in MSSA to QACs of increasing charge state.

\begin{tabular}{cc} 
Compound & MSSA Resistance \\
\hline BAC & $\begin{array}{l}\text { 2-fold at 11 days } \\
\text { 4-fold at 24 days }\end{array}$ \\
DDAC & 2-fold at 10 days \\
CPC & 2-fold at 17 days \\
PQ-12,Bn & 2-fold at 7 days \\
12(3)12 & None over 24 days \\
12(3)2(3)12 & None over 24 days \\
$12(2) 3 \mathrm{~A}(2) 3 \mathrm{~A}(2) 12$ & None over 24 days \\
\hline
\end{tabular}

\section{Conclusion}

Through our studies on the antibacterial activity of various classes of QACs, several key points have become apparent. Amphiphilic structures bearing significant alkyl chains are adept at penetrating membranes of both Gram-positive and Gramnegative bacteria. We have shown that aryl, linear alkyl, cyclic alkyl, and natural product-derived QACs can be prepared in a straightforward manner from economic starting materials, yielding a variety of potent antimicrobial QAC scaffolds. The evolution of QACs has led structures that are capable of eradicating pre-established biofilms in a potent manner. Furthermore, our efforts have led to the finding that bacteria displaying resistance to QACs tend to recognize monocationic conformationally-rigid structures, while QACs possessing higher charge state and conformational flexibility tend to escape such resistance mechanisms. Future efforts will entail the chemical probing of QAC resistance machinery in order to gain a better understanding of the evolution of this system and to develop disinfectants that remain efficacious and one step ahead of pathogenic microbial life.

copper(II) sulfate pentahydrate $(0.037 \mathrm{~g}, 0.15 \mathrm{mmol})$ The mixture was stirred at room temperature for 18 hours. The reaction mixture was quenched with cold water then filtered. The filtered solid was rinsed with ammonium hydroxide (2 M) to afford the desired product $\mathbf{P m T}_{\mathbf{1 0}}(0.833 \mathrm{~g}, 99.8 \%)$ as a tan solid.

1.2. 1-dodecyl-3-(1-decyl-1H-1,2,3-triazol-4-yl)pyridine-1-um bromide $\left(\mathrm{PmT}_{10}-12\right)$

To a solution of 3-(1-decyl-1H-1,2,3-triazol-4-yl)pyridine-1um bromide $(0.201 \mathrm{~g}, 0.700 \mathrm{mmol})$ in acetonitrile $(1.75 \mathrm{~mL}, 33.5$ mmol) was added dodecylbromide $(0.680 \mathrm{~mL}, 2.81 \mathrm{mmol})$. The mixture was heated to reflux for 19 hours, then concentrated under reduced pressure and filtered. The filtered solid was rinsed with cold cyclohexane to afford the desired product $\mathbf{P m T} \mathbf{T}_{\mathbf{1 0}} \mathbf{- 1 2}$ $(0.226 \mathrm{~g}, 60.4 \%)$ as a tan-brown solid. 


\section{References}

1. Tezel, U.; Pavlostathis, S.G. In Antimicrobial Resistance in the Environment; Keen, P.L.; Monforts, M.H., Ed.; John Wiley \& Sons, Inc.: New York, 2011, Vol. 1, pp 349-387.

2. Centers for Disease Control and Prevention. Antibiotic Resistance Threats in the United States; 2013, pp 1-113.

3. Davey, M.E.; O’Toole, G.A. Microbiol. Mol. Biol. Rev. 2000, 64, 847-867.

4. Kostakioti, M.; Hadjifrangiskou, M; Hultgren, S.J. Cold Spring Harb. Perspect. Med. 2013, 3, a010306.

5. Hall-Stoodley, L.; J.W. Costerton, J.W.; Stoodley, P. Nat. Rev. Microbiol. 2004, 2, 95-108.

6. Jennings, M.C.; Minbiole, K.P.C.; Wuest, W.M. ACS Infect. Dis. 2015, 289-303.

7. Čerňáková, M.; Koštálová D. Folia Microbiol. 2002, 47, 375-378.

8. Böttcher, T.; Kolodkin-Gal, I.; Kolter, R.; Losick, R.; Clardy, J. J. Am. Chem. Soc. 2013, 135, 2927-2930.

9. Jennings, M.C.; Ator, L.E.; Paniak, T.J.; Minbiole, K.P.C.; Wuest, W.M. ChemBioChem. 2014, 2211-2215.

10. a) Jacobs, W.A. J. Exp. Med. 1916, 23, 563-568; b) Jacobs, W.A. J. Exp. Med. 1916, 23, 569-576; c) Jacobs, W.A. J. Exp. Med. 1916, 23, 577- 599 .

11. Domagk, G. Dtsch. Med. Wiss. 1935, 61, 829-832.

12. Israelachvili, J. Fluid-Like Structure and Self-Assembling Systems: Micelles, Bilayers and Biological Membranes. In Intermolecular and Surface Forces; Academic Press: San Diego, CA, 1991; p 341.

13. LaDow, J. E.; Warnock, D. C.; Hamill, K. M.; Simmons, K. L.; Davis, R. W.; Schwantes, C. R.; Flaherty, D. C.; Willcox, J. A.; Wilson-Henjum, K.; Caran, K. L.; Minbiole, K. P. C.; Seifert, K. Eur. J. Med. Chem. 2011, 46, 4219-4226.

14. Methods for Dilution Antimicrobial Tests for Bacteria that Grow Aerobically; Approved Standard; CLSI Document M07-A9: Wayne, PA., 2012, Vol. 32 No. 2.

15. Grenier, M. C.; Davis, R. W.; Wilson-Henjum, K. L.; La-Dow, J. E.; Black, J. W.; Caran, K. L.; Seifert, K.; Minbiole, K. P. C. Bioorg. Med. Chem. Lett. 2012, 22, 4055-4058.

16. Dinis-Oliveria, R. J.; Duarte, J. A.; Sánchez-Navarro, A.; Remião, F.; Bastos, M. L.; Carvalho, F. Crit. Rev. Toxicol. 2008, 38, 13-71.

17. Ator, L. E.; Jennings, M. C.; McGettigan, A. R.; Paul, J. J.; Wuest, W. M.; Minbiole, K. P. C. Bioorg. Med. Chem. Lett. 2014, 24, 3706-3709.

18. Gamsey, S.; Miller, A.; Olmstead, M. M.; Beavers, C. M.; Hirayama, L. C.; Pradhan, S.; Wessling, R. A.; Singaram, B. J. Am. Chem. Soc. 2007, 129, 1278-1286.

19. Jennings, M.C.; Buttaro, B.A.; Minbiole, K.P.C.; Wuest, W.M. ACS. Infect. Dis. 2015, 1, 304-309.
20. Black, J. W.; Jennings, M. C.; Azarewicz, J.; Paniak, T. J.; Grenier, M. C.; Wuest, W. M.; Minbiole, K. P. C. Bioorg. Med. Chem. Lett. 2014, 24, 99-102.

21. Imam, T.; Devinsky, F.; Lacko, I.; Mlynarcik, D.; Krasnec, L. Pharmazie. 1983, 38, 308-310.

22. Zana, R.; Benrraou, M.; Rueff, R. Langmuir. 1991, 7, 1072-1075.

23. El Achouri, M.; Bensouda, Y.; Gouttaya, H. M.; Nciri, B.; Perez, L.; Infante, M. R. Tenside Surf. Det. 2001, 38, 208-215.

24. Mao, X.; He, S.; Li, J.; Tang, C.; Yang, X.; Wang, B.; Feng, Y. Chin. J. Synth. Chem. 2011, 2, 180.

25. Kuperkar, K.; Modi, J.; Patel, K. J. Surfact. Deterg. 2012, 15, 107-115.

26. Baidya, M..; Horn, M.; Zipse, H.; Mayr, H. J. Org. Chem. 2009, 74, 7157-7164.

27. Shibagaki, M.; Matsushita, H.; Shibata, S.; Saito, A.; Tsujino, Y.; Kaneko, H. Heterocycles. 1982, 19, 1641-1645.

28. Joyce, M.D.; Jennings, M.C.; Santiago, C.N.; Fletcher, M.H.; Wuest, W.M; Minbiole, K.P.C. J. Antibiotics. 2016, in press.

29. Paniak, T.J.; Jennings, M.C.; Shanahan, P.C.; Joyce, M.D.; Santiago, C.N.; Wuest, W.M.; Minbiole, K.P.C. Bioorg. Med. Chem. Lett. 2014, 24, 5824-5828.

30. Garrison, A.T.; Bai, F.; Abouelhassan, Y.; Paciaroni, N.G.; Jin, S.; Huigens, R.W. RSC Adv. 2015, 5, 1120-1124.

31. Mitchell, M.A.; Iannetta, A.A.; Jennings, M.C.; Fletcher, M.H.; Wuest, W.M.; Minbiole, K.P.C. ChemBioChem. 2015, 16, 22992303.

32. Marafino, J. N.; Gallagher. T. M.; Barragan, J.; Volkers, B. L.; LaDow, J. E.; Bonifer, K.; Fitzgerald, G.; Floyd, J. L.; McKenna, K.; Minahan, N. T.; Walsh, B.; Seifert, K.; Caran, K. L. Bioorg. Med. Chem. 2015, 23, 3566-3573.

Notes

This work was funded by a University City Science Center QED Proof of Concept Grant (Grant S1403 to WMW), a Research Corporation Grant Multi-Investigator Cottrell College Science Award (MICCSA 10709 to KPCM, KS, and KLC), the National Science Foundation (NSF-REU CHE-1062629 and CHE-0754521), as well as by Temple, Villanova, and James Madison Universities. MCJ acknowledges a National Science Foundation Pre-Doctoral Fellowship (DGE1144462).

\section{Supplementary Material}

Supplementary material containing full biological data and procedures, and compound preparation and characterization for all new compounds, may be found online at 


\section{Graphical Abstract}

To create your abstract, type over the instructions in the template box below.

Fonts or abstract dimensions should not be changed or altered.

\section{From Antimicrobial Activity to Mechanism of Resistance: \\ The Multifaceted Role of Simple Quaternary Ammonium}

\section{Compounds in Bacterial Eradication}

Kevin P.C. Minbiole, * Megan C. Jennings, Laura E. Ator,

Jacab W. Black, Melissa C. Grenier, Jade E. LaDow, Kevin L.

Caran, Kyle Seifert, and William M. Wuest*

Department of Chemistry, Villanova University 800 Lancaster Ave, Villanova, PA 19085

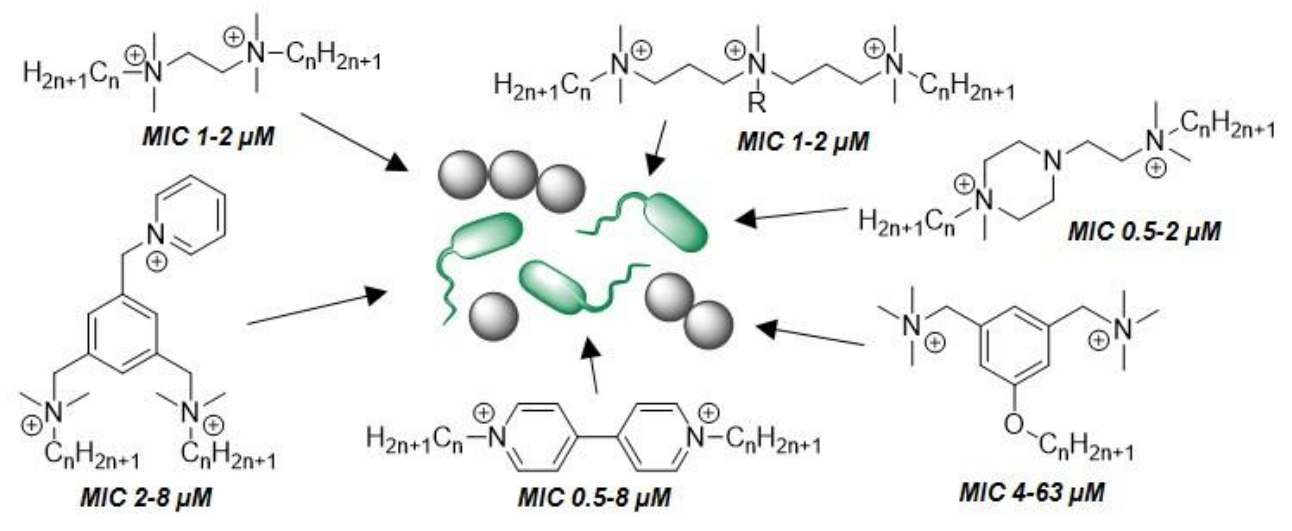

